\title{
The Effect of Balloon Dilation at the Vallecular Using Videofluoroscopic Swallowing Study on Patient Who Has a Dysphagia
}

\author{
Yong Kyun Kim, MD, Min Tae Kim, MD, Seong Kyun Kim, MD
}

Department of Physical Medicine and Rehabilitation, Myongi Hospital, Kwandong University College of Medicine, Goyang, Korea

\begin{abstract}
Authors have previously experienced the effect of balloon dilation at the vallecular by utilizing the videofluoroscopic swallowing study (VFSS) and the urethral catheter to physically stretch and spread in the direction of the posterior inferior towards the patients who have claimed for dysphagia symptoms due to epiglottic dysfunction. A 72-year-old male patient has been diagnosed with rectal cancer and have been treated with an ileocolostomy after the intubation. After the removal of tracheal intubation, the patient complained of dysphagia. Foods and drinks could not be transmigrated into the esophagus due to the inability of the epiglottis to bend backward in the direction of posterior inferior on VFSS. The epiglottis was physically stretched and spread in the direction of posterior inferior by utilizing the balloon attached to a urethral catheter. After stretching and spreading the epiglottis in the direction of posterior inferior, the bolus remaining in the epiglottic vallecula was decreased. For a patient who is experiencing dysphagia due to an epiglottis disorder, it seems that an epiglottis balloon dilation supported by VFSS and a urethral catheter may be appropriate for the treatment of dysphagia symptoms.
\end{abstract}

Keywords Epiglottis, Dysphagia, Balloon dilation

\section{INTRODUCTION}

Dysphagia can cause aspiration pneumonia and dehydration, with continuous dysphagia causing even death

Received December 19, 2011; Accepted July 18, 2012

Corresponding author: Min Tae Kim

Department of Physical Medicine and Rehabilitation, Myongji Hospital, Kwandong University College of Medicine, 55 Hwasu-ro 14beon-gil, Deokyang-gu, Goyang 412-826, Korea

Tel: +82-31-810-6450, Fax: +82-31-810-5259, E-mail: kmt137@hanmail. net

(c) This is an open-access article distributed under the terms of the Creative Commons Attribution Non-Commercial License (http://creativecommons. org/licenses/by-nc/3.0) which permits unrestricted noncommercial use, distribution, and reproduction in any medium, provided the original work is properly cited.

Copyright $\odot 2013$ by Korean Academy of Rehabilitation Medicine in come cases [1]. Epiglottis has a function of preventing inhalation of foods in the airways, and hence, is known as the most important oral structure associated with dysphagia. In the case that epiglottis is displaced to the posterior, dysfunction may occur in the epiglottis, in which case, the epiglottis is not bent back to the posterior inferior causing impediment for swallowing; yet, a special treatment method is not known to be available [2]. Through videofluoroscopic swallowing study (VFSS), we confirmed the dysfunction of the epiglottis with patients complaining of dysphagia, as epiglottis fails to be bent in the direction of posterior inferior, and experienced a treatment by physically stretching and spreading the epiglottis in the direction of posterior inferior, using a urethral catheter, which is reported herein together with 
a literature consideration.

\section{CASE REPORT}

A 72-year-old male patient with stomach cancer and S-phase colon cancer received ileostomy along with gastrectomy and proctocolectomy for cancer removal, followed by concentrated observation treatment in the intensive care unit, during which, dyspnea occurred suddenly. After intubation and artificial venting by a respirator, respiration-related symptoms were improved and spontaneous respiration became possible after removing the respirator. However, dysphagia occurred, and he was admitted by the department rehabilitation medicine in the present hospital for comprehensive rehabilitation treatment. Although the patient did not have a past history of high blood pressure or diabetes, he had a history of drinking alcohol 4 times a week and smoking for 40 pack years, and was taking warfarin due to atrial fibrillation. He had a history of receiving percutaneous transluminal coronary angioplasty due to angina pectoris. According to a physical exam, no particular finding was observed other than weakness of the whole body.

The patient had received a continuous nutrition supply through total parenteral nutrition for more than 2 months since the first onset, and changed nutrition supply to tube feeding through nasogastric tubing after being hospitalized by the present hospital, followed by continued exercise promoting tongue's posterior movement and laryngeal elevation through occupational therapy. After that, VFSS was conducted to analyze the possibility of oral nutrition, during which, a large quantity of remnant epiglottis vallecula was observed without, however, a finding for aspiration so that nasogastric tubing was removed and oral nutrition was attempted through a compensation technique. Since then, however, there was a complaint of continued dysphagia during eating, and esophagogastroduodenoscope (EGD) examination was conducted through a consultation with the division of gastroenterology for a detailed cause analysis. From the EGD examination, no finding of mechanical closure of esophagus or stomach was observed. For a more accurate diagnosis, esophageal pressure manometry was conducted, which indicated normal findings for both the length of the upper and lower esophagus sphincter muscles or the resting pressure, as well as coordination of the esophagus during swallowing and fluid changes. Fol- lowing that, VFSS was conducted again and a comparative analysis with the previous study images was made. The result of which led to a judgment that epiglottis was not bent back toward posterior inferior so that foods did not pass to the esophagus, Hence, an intervention was conducted where the epiglottis was physically stretched and spread in the direction of posterior inferior, using a urethral catheter. Intervention involved first implementation of the swallowing test, positioning a $16 \mathrm{~F}$ urethral catheter in the epiglottis vallecula, and subsequently expanding the balloon, as well as stretching and spreading of the epiglottis for 1 minute using about $5 \mathrm{~mL}$ of a contrast medium for balloon expansion; followed by rest for 10 seconds, which were repeated for a total of 3 times for the treatment (Fig. 1). Thereafter, swallowing test was again conducted to make judgment on the treatment effects (Figs. 2, 3).

Since then, an improvement in dysphagia symptoms was indicated with the ability of eating semisolid foods, and stretching and spreading treatment of the epiglottis was continued with progress observation. In addition, the use of a urethral catheter was accompanied by re-implementation of VFSS once a week. As a result, improvement was made to the extent of being able to eat solid foods, allowing discharge from the hospital.

\section{DISCUSSION}

For the treatment of dysfunction of the cricopharyngeal muscle through VFSS, balloon dilation using a urethral

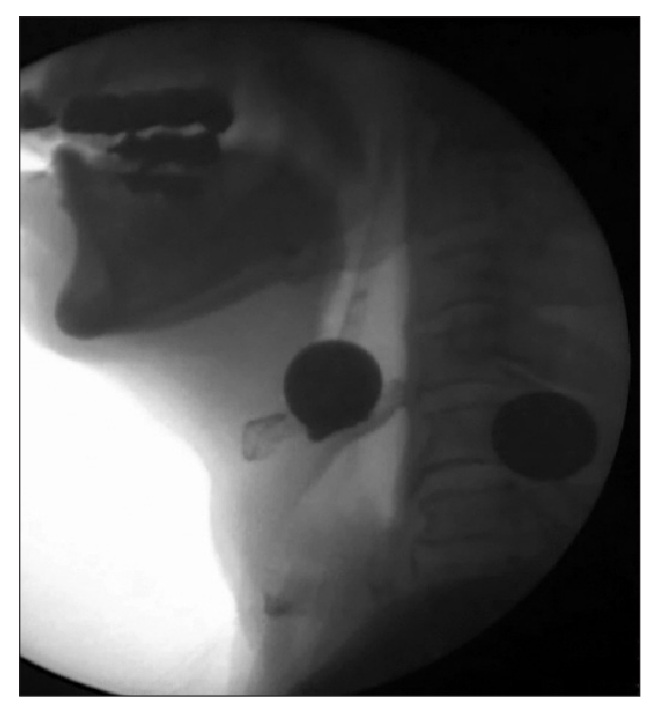

Fig. 1. Balloon dilatation at the vallecula. 

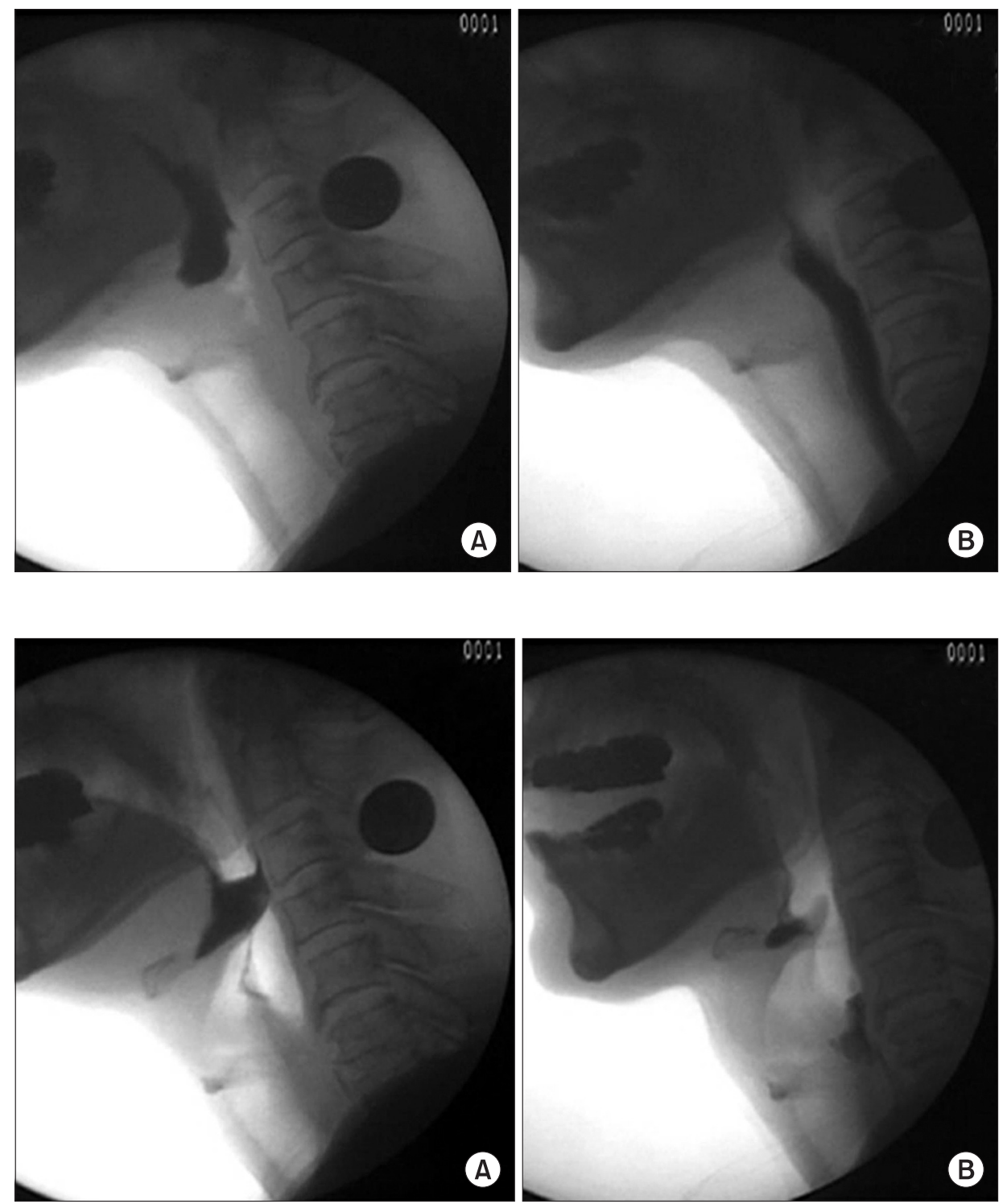

Fig. 2. During-swallow bent back of epiglottis before (A) and after (B) balloon dilation. catheter is known to be useful for a reduction of the pharyngeal transmigration time of foods and the amount of pharyngeal remnants [3]. Particularly, balloon dilation is known to be capable of providing help in obtaining information needed for diagnosing dysphagia, as well as in treating primary dysfunction of the cricopharyngeal muscle $[4,5]$.

However, changes in the esophagus muscle's cross section observed after balloon dilation are known not to be related to the improvement in dysphagia symptoms [6]. Symptoms are known to recur frequently upon longterm follow-up observations with many patients treated for dysphagia with balloon dilation; hence, operation methods are recommended as well in the case where response to a conservative treatment is minimal [7]. Jung et al. [8] compared the effects of sphincter muscle excision and balloon dilation in children's esophageal achalasia to find that the effects of conservative treatment and operation were similar in the case above 6 years of age, and recommended the balloon dilation as a treatment that can be attempted first for dysphagia.

Although botulinum toxin injection is also recommended for muscles with thickening of cricopharyngeus, usefulness for the effects is still controversial [1].

While epiglottis is a structure blocking inhalation of foods in airways and has a close linkage with major complications of dysphagia $[9,10]$, studies related to epiglottis are rarely known as compared with dysfunction of the cricopharyngeal muscle.

Since the catheter moves past the nasal cavity dur- 
Table 1. Change of laryngeal elevation, pharyngeal transit time, vallecular remnant after balloon dilation at the vallecular

\begin{tabular}{lrc}
\hline \multicolumn{1}{c}{ Parameter } & Pre & Post \\
\hline Laryngeal elevation $(\mathrm{mm})$ & 17 & 21 \\
Pharyngeal transit time $(\mathrm{ms})$ & 6000 & 800 \\
Vallecular remnant $\left(\mathrm{mm}^{2}\right)$ & 36 & 6 \\
\hline
\end{tabular}

ing execution of intervention as a side effect of balloon stretching and spreading, there may be a sense of comfort and temporary pain in the patient during the examination. However, in the case of the present patient, intervention cooperation was well executed without complaints of great discomfort, and no serious side effects or recurrence were observed up to the last follow-up observation.

The present report is a case where balloon is directly used on the epiglottis for passive stretching and spreading with a dysphagia patient due to lowered exercise of the epiglottis, unlike general balloon dilation used for dysphagia patients due to the dysfunction of cricopharyngeal muscle. After stretching and spreading epiglottis in the direction of posterior inferior, the amount of epiglottis remnant was decreased, showing finding that laryngeal elevation and pharyngeal transit time were improved (Table 1). Hence, improvement of symptoms by balloon stretching and spreading of epiglottis has been experienced in a patient with occurrence of dysphagia, using VFSS and urethral catheter. This case is reported herein along with literature considerations.

\section{CONFLICT OF INTEREST}

No potential conflict of interest relevant to this article was reported.

\section{REFERENCES}

1. Kim HJ, Yun DH, Kim SH, Kim DY, Kim HS, Kim HJ. Endoscopic botulinum toxin injection for the treat- ment of dysphagia caused by cricopharyngeal hypertonicity: a case report. J Korean Acad Rehabil Med 2006;30:398-401.

2. Garon BR, Huang Z, Hommeyer M, Eckmann D, Stern GA, Ormiston C. Epiglottic dysfunction: abnormal epiglottic movement patterns. Dysphagia 2002;17:5768.

3. Kim JC, Kim JS, Jung JH, Kim YK. The effect of balloon dilatation through video-fluoroscopic swallowing study (VFSS) in stroke patients with cricopharyngeal dysfunction. J Korean Acad Rehabil Med 2011;35:23-6.

4. Solt J, Bajor J, Moizs M, Grexa E. Primary cricopharyngeal achalasia and its dilatation with balloon catheter. Orv Hetil 2000;141:2287-92.

5. Solt J, Bajor J, Moizs M, Grexa E, Horvath PO. Primary cricopharyngeal dysfunction: treatment with balloon catheter dilatation. Gastrointest Endosc 2001;54:76771.

6. Sinn DH, Choi YS, Kim JH, Kim ER, Son HJ, Kim JJ, et al. Change in cross-sectional area of esophageal muscle does not correlate with the outcome of achalasia after pneumatic balloon dilatation. J Gastroenterol Hepatol 2010;25:539-43.

7. Alderliesten J, Conchillo JM, Leeuwenburgh I, Steyerberg EW, Kuipers EJ. Predictors for outcome of failure of balloon dilatation in patients with achalasia. Gut 2011;60:10-6.

8. Jung C, Michaud L, Mougenot JF, Lamblin MD, Philippe-Chomette P, Cargill G, et al. Treatments for pediatric achalasia: Heller myotomy or pneumatic dilatation? Gastroenterol Clin Biol 2010;34:202-8.

9. Garon BR, Huang Z, Hommeyer M, Eckmann D, Stern GA, Ormiston C. Epiglottic dysfunction: abnormal epiglottic movement patterns. Dysphagia 2002;17:5768.

10. Han TR, Bang MS, Paik NJ, Jeon JY, Kim SJ, Lee HJ. Digital image motion analysis of the pharyngeal movement during swallowing in dysphagia patients. J Korean Acad Rehabil Med 2002;26:693-8. 